NISTIR 7645

\title{
Symbols Representing Biometrics in Use
}

\author{
Yee-Yin Choong \\ Mary Theofanos \\ Brian Stanton \\ Patrick Hofmann
}

National Institute of Standards and Technology • U.S. Department of Commerce 
NISTIR 7645

\section{Symbols Representing Biometrics in Use}

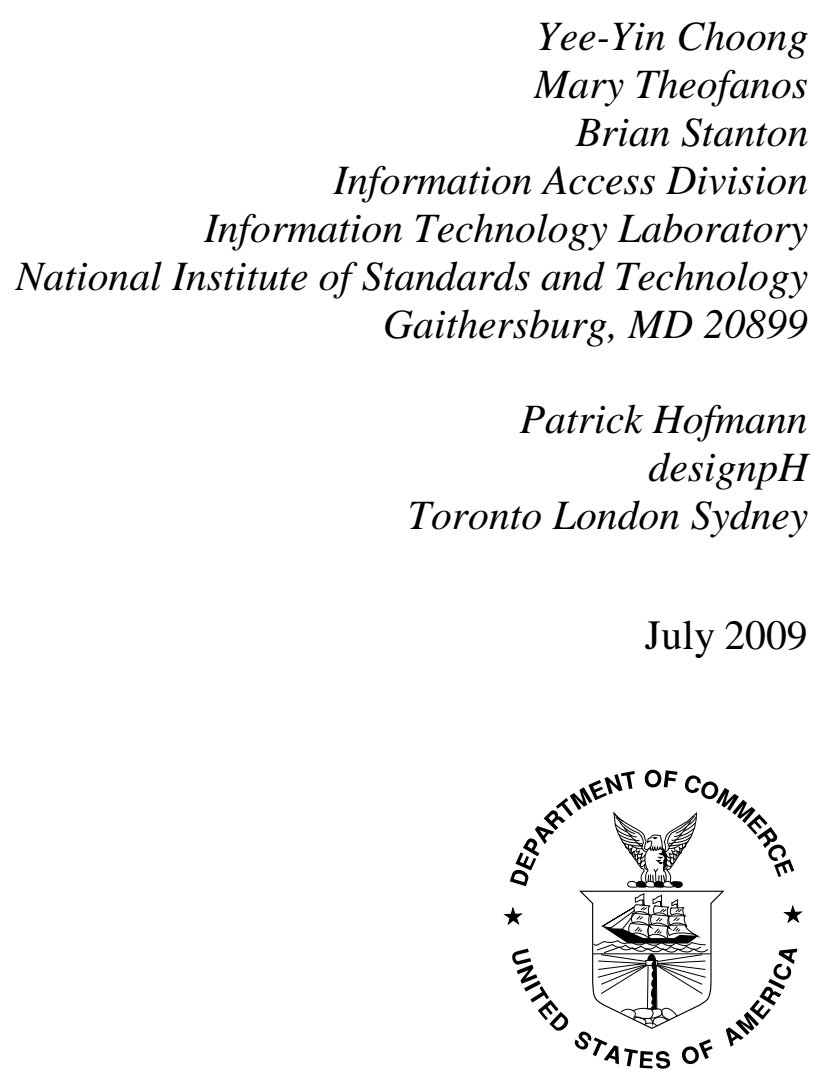

U.S. Department of Commerce Gary Locke, Secretary

National Institute of Standards and Technology Patrick D. Gallagher, Deputy Director 


\section{$\underline{\text { Table of Contents }}$}

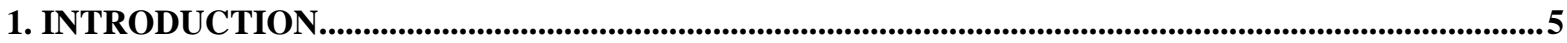

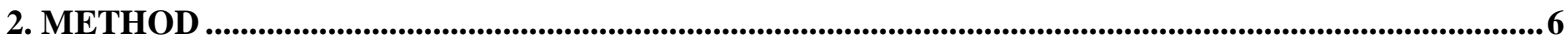

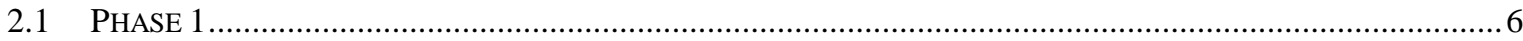

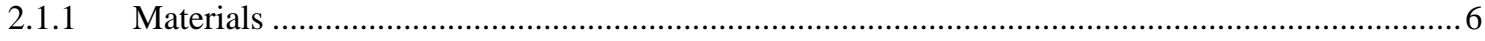

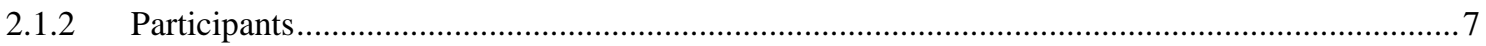

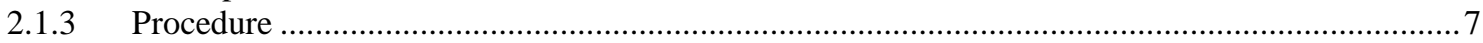

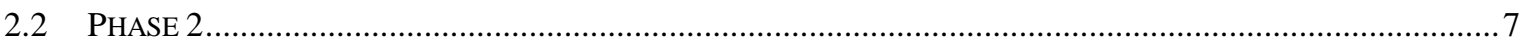

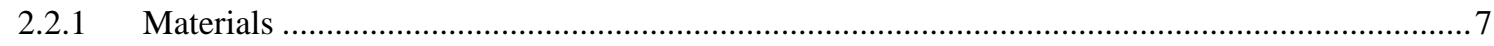

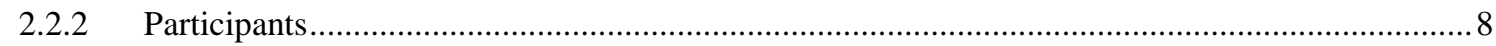

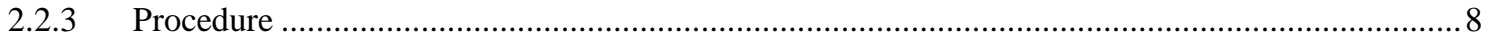

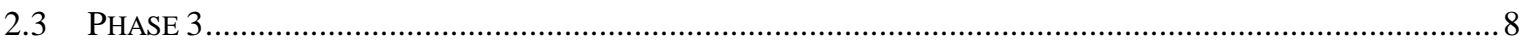

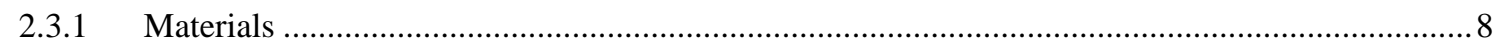

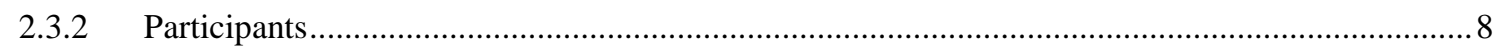

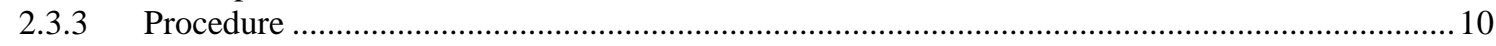

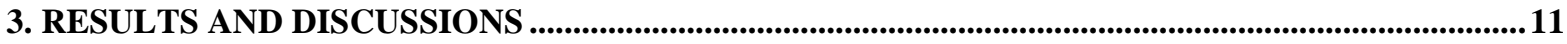

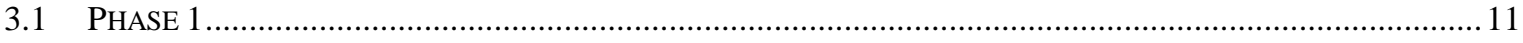

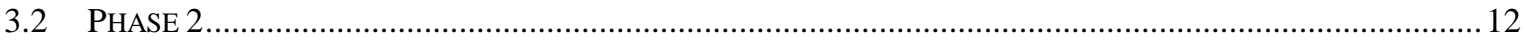

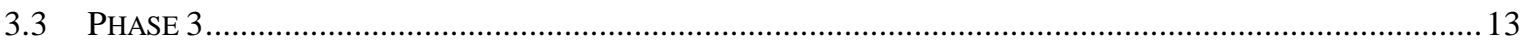

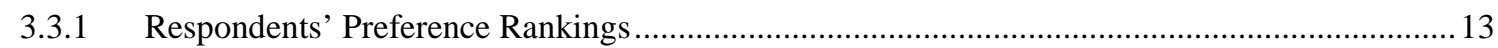

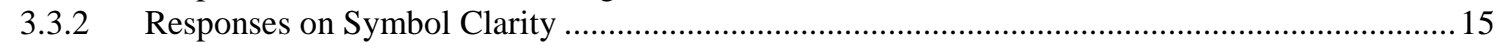

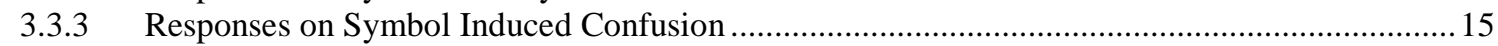

3.3.4 Responses on Confidence in Symbol Recognition.......................................................... 16

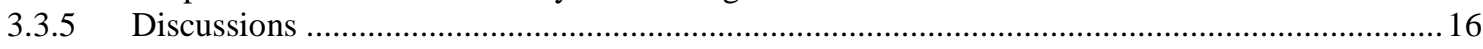

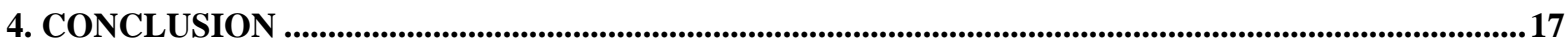

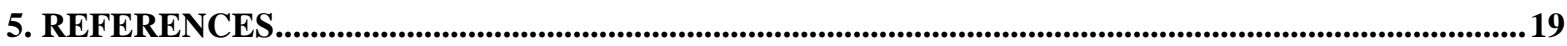

APPENDIX A: PHASE 3 - EMAIL TO INVITE SURVEY PARTICIPANTS..........................................20

APPENDIX B: PHASE 3 - EXAMPLE OF BIOMETRICS SYMBOL SURVEY ................................21 


\section{$\underline{\text { List of Figures }}$}

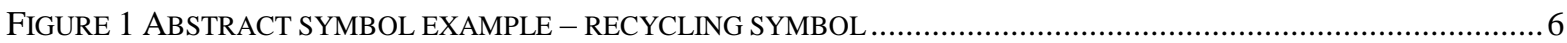

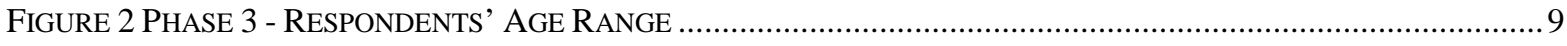

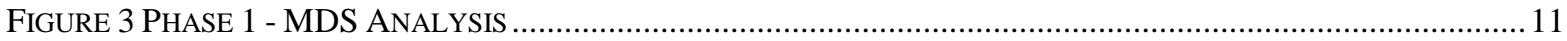

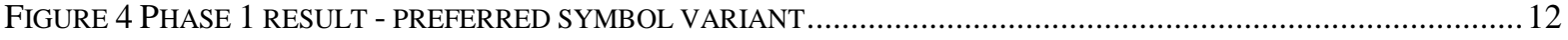

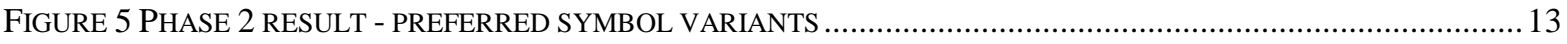

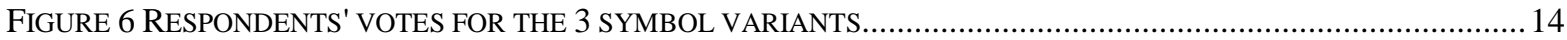

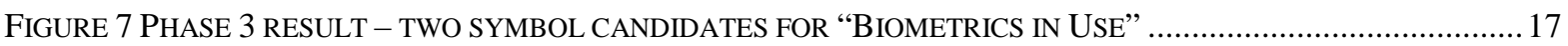

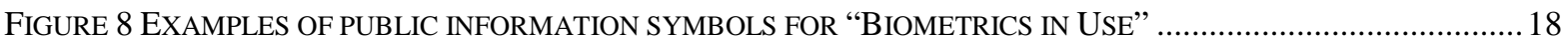

FIGURE 9 EXAMPLES OF ALERTING SYMBOLS FOR "BIOMETRICS IN USE" ................................................... 18 


\section{INTRODUCTION}

During the InterNational Committee for Information Technology Standards (INCITS) M1 task group meetings in 2008, it was recognized that there is a need for a symbol to represent that any biometrics (fingerprint, iris, photo, etc.) are in use. The symbol is to be used to indicate that biometric collection may be in use, for example, to indicate to the public that their photo may be taken or their fingerprints may be scanned. The symbol should be independent of any specific biometric collection processes. It will also need to be generic such that it is applicable to all of the modalities including iris, face, fingerprint, voice, vein, and temperature and any modalities that may be developed in the future.

There is no existing symbol with the intended meaning either in ISO 7001 [1] for public information symbols, ISO/IEC 13251 [2] for office equipment, nor in existing ISO standards concerning safety symbols for various applications (ISO 3864-1 [3], ISO 7010 [4], ISO 7010 - Amendment 1 [5], ISO 7010 - Amendment 2 [6], ISO 7010 - Amendment 3 [7], ISO 7010 - Amendment 4 [8], ISO 6309 [9], ISO 20712-1 [10]). It is apparent that such symbol needs to be developed to convey the concept of biometrics in use to people.

Initially, the intent was to develop a symbol to alert the public that biometrics are in use. The National Institute of Standards and Technology (NIST) Biometrics Usability group developed a set of symbol variants and performed a study to evaluate those variants. Each variant was bordered with the cautionary triangle to convey the alert. The variants were evaluated with representative audience in phases. In the first phase, 10 variants were evaluated with 49 participants using an interview technique. In the second phase, five variants (including one variant chosen from phase 1 and four new symbol variants) were evaluated with another 50 participants using the same interview technique as in phase 1.

Two variants were identified as plausible candidates for the intended meaning as the results from the first two phases. Those two variants were socialized during the ISO/IEC JTC 1/SC 37 working group meeting, WG 6 on Cross-Jurisdictional and Societal Aspects, 20-23 January 2009 in Hawaii. Comments from ISO members regarding the two variants indicated that the intended meaning should be informative, rather than alerting, to the general public about biometrics in use. ISO members were concerned that the triangular border which implies warning may cause the public perceive biometrics collection as alarming and harmful. Based on the comments, another set of three variants without the triangular border was developed and a phase 3 evaluation has been conducted with an on-line survey. The objective of the survey was to reach out an audience with broader backgrounds such as age, occupations, and ethnicity. 


\section{METHOD}

This study was conducted in three phases. The details of each phase are described in the following sections.

\subsection{PHASE 1}

\subsubsection{Materials}

Initially, a set of twenty-four, black-and-white symbol variations were developed, each bordered with a cautionary triangle. The purpose of the symbols is to provide general indication to the public that any biometrics may be in use. Due to its objective of being generic, the design concept was to create symbols that may be abstract to portrait the generic nature, yet can be perceived uniquely and will be learned and remembered. Such design concept can be illustrated by the well known standard recycling symbol (ISO 7000-1135 in ISO/IEC 13251 [2]), in Figure 1, which was designed to be memorable but abstract. The recycling symbol has been learned and recognized by the general public.

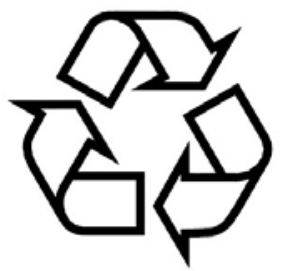

Figure 1 Abstract symbol example - recycling symbol

Three experts at NIST reviewed and reduced the set to 10 symbol variants (Table 1) based on information available to NIST experts regarding the intended purpose of the symbol at the time of the review.

Table 1 Symbol Variants for Phase 1

variant 1-1




\subsubsection{Participants}

The recruitment of participants was cautioned to exclude anyone whose job or background is related to biometrics to eliminate possible biases. Forty-nine NIST employees volunteered to participate in phase 1 evaluation.

\subsubsection{Procedure}

An one-on-one interview technique was used in phase 1. All interviews were conducted in the participants' office. The interviewer provided each participant with the background of the study, explaining that we were designing symbols to indicate that biometrics are in use in the vicinity, similar to the warning sign of surveillance video in use in the building where his/her office is. The interviewer further explained that symbols of this kind are generally learned, for example, the recycling symbol (Figure 1) that cannot be recognized without learning it first. The symbol we are trying to develop will need to be learned but should be memorable and linked to biometrics. It will also need to be generic such that it is applicable to all of the biometric modalities.

The interviewer informed each participant that there would be 10 symbol variants (Table 1) and asked the participant to order them from the one most appropriate for a general symbol indicating biometrics in use to the one least appropriate. Each participant received a stack of 10 sheets of paper, each paper with only one symbol variant printed on it. The presentation sequence of the 10 variants was randomized for each participant to eliminate any sequence effects. There was no time limit on ordering the symbol variants. The participants indicated to the interviewer when they finished the task. Any comments made by the participants were noted by the interviewer.

\subsection{PHASE 2}

\subsubsection{Materials}

In phase 2, four additional symbols were developed and evaluated with the most preferred symbol variant (variant 1-6 in Table 1) from phase 1. As the variants in phase 1 were mainly abstract, it was the intention in phase 2 to test symbols with a range of abstract and concrete concepts. Total of five variants were evaluated, as shown in Table 2.

Table 2 Symbol Variants for Phase 2

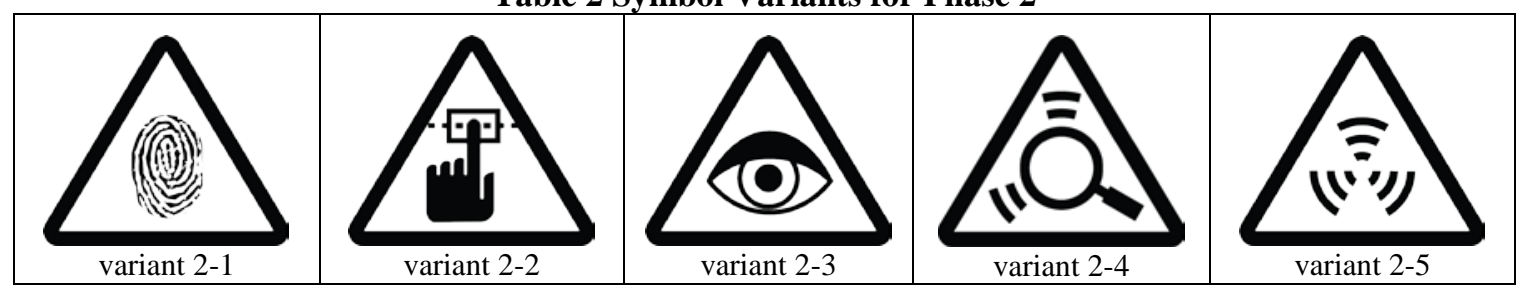




\subsubsection{Participants}

Same as in phase 1, the recruitment of participants was cautioned to exclude anyone whose job or background is related to biometrics so that possible biases were eliminated. The recruitment also excluded anyone who participated in phase 1. Fifty NIST employees volunteered to participate in phase 2 evaluation.

\subsubsection{Procedure}

The procedure was the same as in phase 1 . All interviews were conducted in the participants' office. The interviewer provided each participant with the same information regarding the study and the context of use as given in phase 1.

The interviewer informed each participant that there would be five symbol variants (Table 2) and asked the participant to order them from the one most appropriate for a general symbol indicating biometrics in use to the one least appropriate. Each participant received a stack of five sheets of paper, each paper with only one symbol variant printed on it. The presentation sequence of the five variants was randomized for each participant to eliminate any sequence effects. There was no time limit on ordering the symbol variants. The participants indicated to the interviewer when they finished the task. Any comments made by the participants were noted by the interviewer.

\subsection{PHASE 3}

\subsubsection{Materials}

Three symbol variants were developed without the warning triangular borders: two variants were the results from phase 2 and one was new. Those three variants (Table 3) were evaluated in phase 3 .

Table 3 Symbol Variants for Phase 3

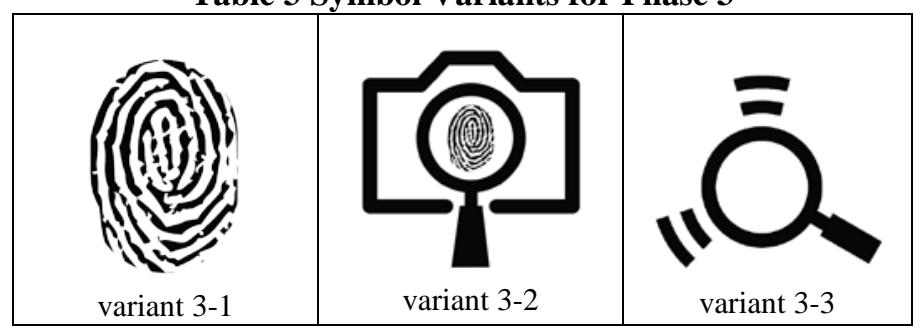

\subsubsection{Participants}

In order to reach out to a broader audience, an invitation email of a survey was sent to a nation-wide user pool of about 12,000 people with various backgrounds such as gender, age, education, occupation, and ethnicity. The email was carefully written in plain English to 
ensure that all readers with different backgrounds can understand it. The email was finalized (Appendix A) after an 8-year-old, $2^{\text {nd }}$ grader proofread it with complete comprehension. If any of the email recipients decided to participate, he/she would access the survey via an URL provided within the email.

\subsubsection{Demographics}

Within a two-week span, total of 725 respondents took the survey. There were 520 females, 202 males, and three respondents who chose not to identify their genders. The age of the respondents ranged from 18 to above 75 (Figure 2), with majority (76.7\%) between 25 and 54.

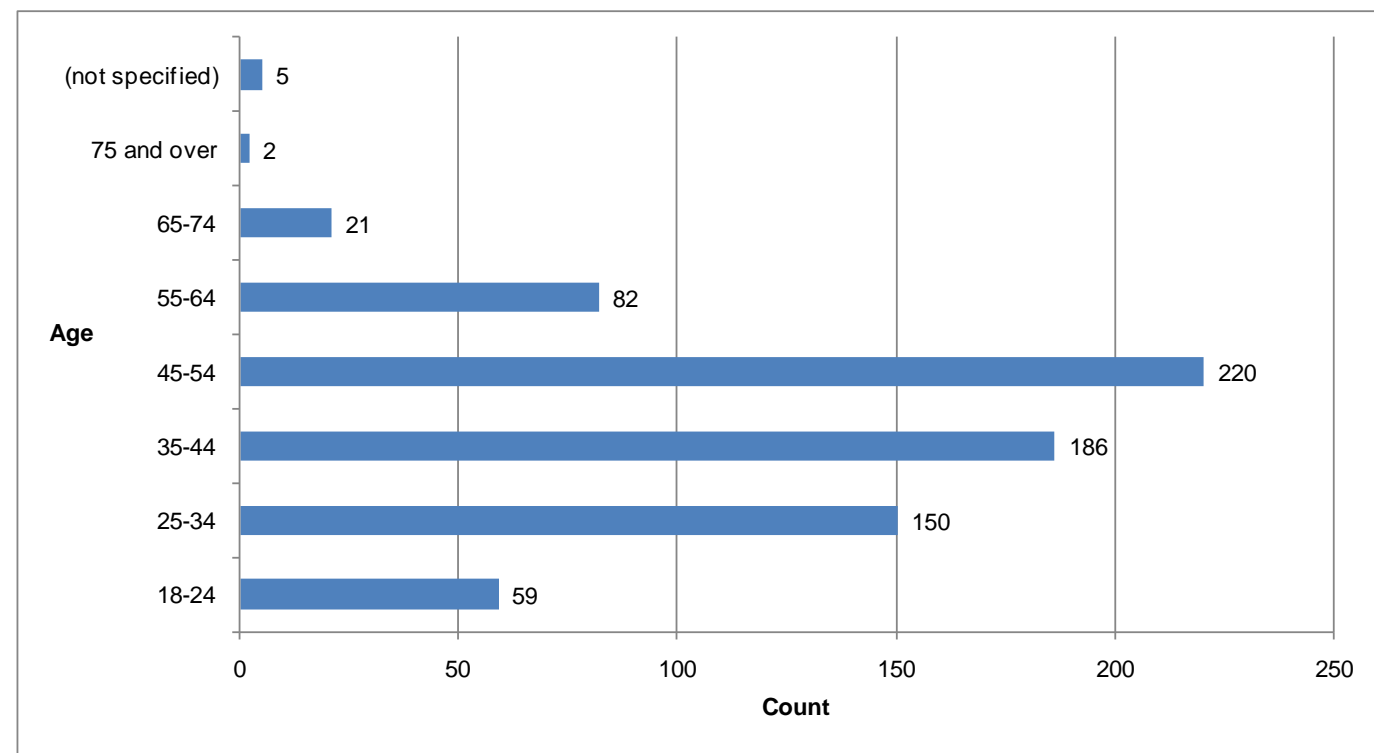

Figure 2 Phase 3 - Respondents’ Age Range

The respondents' ethnic backgrounds were diverse with majority white Americans. The respondents' jobs covered a wide range of occupations. The respondents' ethnic backgrounds and occupations are summarized in Table 4.

Table 4 Phase 3 - Respondents' Ethnicity and Occupations

\begin{tabular}{|l|r||l|r|}
\hline \multicolumn{1}{|c|}{ Ethnic Backgrounds } & \multicolumn{1}{c|}{ Count (\%) } & \multicolumn{1}{c|}{ Occupations } & \multicolumn{1}{c|}{ Count (\%) } \\
\hline White American & $542(74.8 \%)$ & Administrative support worker & $94(13.0 \%)$ \\
\hline African American & $85(11.7 \%)$ & Craft worker & $16(2.2 \%)$ \\
\hline Latino American & $34(4.7 \%)$ & Homemaker & $137(18.9 \%)$ \\
\hline American Indian or Alaskan Native & $6(0.8 \%)$ & Laborer & $41(5.7 \%)$ \\
\hline East Asian American & $3(0.4 \%)$ & Professional & $198(27.3 \%)$ \\
\hline South Asian American & $6(0.8 \%)$ & Retired & $61(8.4 \%)$ \\
\hline Native Hawaiian or other Pacific Islander & $1(0.1 \%)$ & Sales worker & $21(2.9 \%)$ \\
\hline Canadian & $2(0.3 \%)$ & Service worker & $40(5.5 \%)$ \\
\hline East Asian & $6(0.8 \%)$ & Student & $42(5.8 \%)$ \\
\hline European & $9(1.2 \%)$ & Technician & $29(4.0 \%)$ \\
\hline
\end{tabular}




\begin{tabular}{|l|r||r|r|}
\hline Latino & $4(0.6 \%)$ & Other & $46(6.3 \%)$ \\
\hline Mediterranean & $3(0.4 \%)$ & & \\
\cline { 1 - 2 } South Asian & $10(1.4 \%)$ & & \\
\cline { 1 - 2 } Other & $14(1.9 \%)$ & & \\
\hline
\end{tabular}

\subsubsection{Procedure}

An on-line survey (Appendix B) ${ }^{1}$ was developed to collect respondents' preferences on the symbol variants for "biometrics in use". The survey also collected respondents' opinions on each symbol variant, concerning its clarity, whether it causes confusion, and the extent that the respondents feel confident to recognize the symbol variant with its intended meaning in the future.

To eliminate any possible biases that may result from the presentation sequence of the three symbol variants evaluated, six variations of the survey were created to account for all possible symbol presentation sequences. All follow-up questions regarding individual symbol variants were arranged in the same sequence as how the variants were presented in the preference question (Question 5 on the survey, Appendix B). For example, in Appendix $B$, it shows survey variation 5 with the symbol presentation sequence: variant 3-1, variant 32, and variant 3-3.

The six survey variations were roughly even-distributed to respondents (Table 5). When the first respondent accessed the on-line survey, the first survey variation was displayed. The second survey variation was displayed to the second respondent, then the third respondent will see survey variation 3 , and so on. The six survey variations followed in sequence and in cycles.

Table 5 Survey Variations Presented to Respondents

\begin{tabular}{|c|c|r|}
\hline $\begin{array}{c}\text { Survey } \\
\text { Variations }\end{array}$ & Sequence & \# of Respondents \\
\hline 1 & variant 3-3, variant 3-1, variant 3-2 & 127 \\
\hline 2 & variant 3-3, variant 3-2, variant 3-1 & 132 \\
\hline 3 & variant 3-2, variant 3-1, variant 3-3 & 114 \\
\hline 4 & variant 3-1, variant 3-3, variant 3-2 & 119 \\
\hline 5 & variant 3-1, variant 3-2, variant 3-3 & 120 \\
\hline 6 & variant 3-2, variant 3-3, variant 3-1 & 113 \\
\hline \multicolumn{2}{|r|}{ Total } & 725 \\
\cline { 2 - 3 } & &
\end{tabular}

${ }^{1}$ The OMB number for this survey is 0693-0043, expiring on 7/31/2009. 


\section{RESULTS AND DISCUSSI ONS}

\subsection{PHASE 1}

After each participant ordered the 10 symbol variants based on his/her preference, the rankings ( 1 to 10 ) were recorded, with " 1 " denoting the most preferred variant and " 10 " the least preferred variant. The preference rankings are summarized in Table 6 for each symbol variant: each cell lists the number of participants who ordered the variant with the specific rank. For example, the top left-most cell indicates that there were 10 participants who ordered variant 1-1 as their most preferred symbol.

Table 6 Counts of participants' rankings for phase 1 symbol variants

\begin{tabular}{|l|r|r|r|r|r|r|r|r|r|r|}
\hline Ranks & \multicolumn{1}{|c|}{ V 1-1 } & \multicolumn{1}{|c|}{ V 1-2 } & \multicolumn{1}{|c|}{ V 1-3 } & \multicolumn{1}{|c|}{ V 1-4 } & V 1-5 & V 1-6 & V 1-7 & V 1-8 & V 1-9 & V 1-10 \\
\hline 1st & 10 & 2 & 2 & 2 & 5 & 9 & 6 & 1 & 6 & 6 \\
\hline 2nd & 5 & 4 & 5 & 2 & 4 & 7 & 6 & 4 & 5 & 7 \\
\hline 3rd & 1 & 6 & 6 & 2 & 2 & 3 & 9 & 7 & 7 & 6 \\
\hline 4th & 3 & 5 & 6 & 8 & 2 & 7 & 4 & 1 & 9 & 3 \\
\hline 5th & 3 & 3 & 5 & 4 & 7 & 5 & 9 & 5 & 0 & 8 \\
\hline 6th & 2 & 10 & 5 & 2 & 4 & 6 & 4 & 10 & 2 & 4 \\
\hline 7th & 3 & 8 & 3 & 4 & 3 & 6 & 7 & 4 & 8 & 3 \\
\hline 8th & 6 & 2 & 10 & 1 & 2 & 2 & 1 & 12 & 7 & 5 \\
\hline 9th & 2 & 6 & 6 & 14 & 8 & 3 & 2 & 2 & 4 & 3 \\
\hline 10th & 14 & 3 & 1 & 10 & 12 & 1 & 1 & 3 & 1 & 4 \\
\hline
\end{tabular}

As the rankings over the 10 symbol variants were ordinal and related (within participants), a non-parametric test, the Friedman's analysis of variance by ranks, was used. Differences in preferences across symbol variants were significant, $\chi^{2}(9)=32.865, p<=0.0001$.

Derived Stimulus Configuration

Euclidean distance model

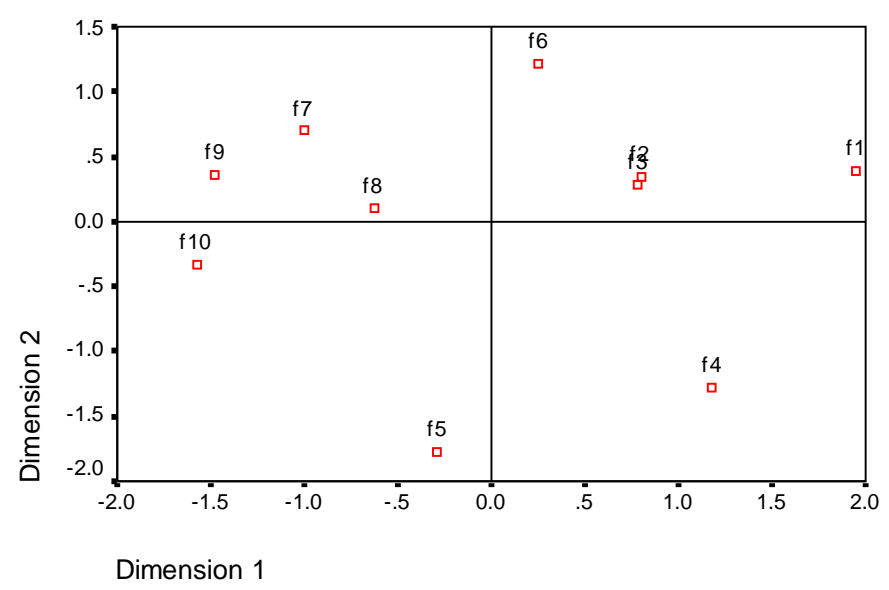

Figure 3 Phase 1 - MDS Analysis 
The Multidimensional Scaling (MDS) method was used to detect any possible underlying dimensions that may explain the similarities or dissimilarities between the preference data collected from the forty-nine participants for those 10 symbol variants in phase 1 . The results of the MDS analysis is shown in Figure 3, where f1to f10 represent symbol variant1-1 to variant $1-10$, respectively.

The vertical dimension (Dimension 2) appears to represent "preference", with higher values for higher preferences. The horizontal dimension (Dimension 1) seems to be a measure of symbol complexity - high numbers show the simple cases and negative ones are more complex. Based on the MDS analysis, one symbol (f6, i.e. variant 1-6), shown in Figure 4, was chosen to be moved forward for phase 2 evaluation as it was scored the highest in preference and on the positive side of symbol complexity.

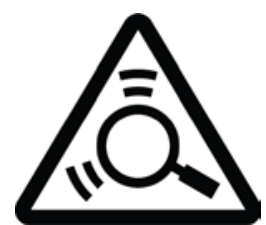

Figure 4 Phase 1 result - preferred symbol variant

\subsection{PHASE 2}

After each participant ordered the five symbol variants based on his/her preference, the rankings ( 1 to 5 ) were recorded, with " 1 " denoting the most preferred variant and " 5 " the least preferred variant. The preference rankings are summarized in Table 7 for each symbol variant: each cell lists the number of participants who ordered the variant with the specific rank.

Table 7 Counts of participants' rankings for phase 2 symbol variants

\begin{tabular}{|l|r|r|r|r|r|}
\hline Ranks & V 2-1 & \multicolumn{1}{|c|}{ V 2-2 } & V 2-3 & V 2-4 & V 2-5 \\
\hline 1st & 20 & 5 & 7 & 16 & 10 \\
\hline 2nd & 11 & 9 & 14 & 12 & 4 \\
\hline 3rd & 5 & 11 & 13 & 9 & 10 \\
\hline 4th & 11 & 14 & 8 & 9 & 5 \\
\hline 5th & 3 & 11 & 8 & 4 & 21 \\
\hline
\end{tabular}

Friedman's analysis of variance by ranks was used on these five symbol variants and their rankings received from the participants. Differences in preferences across symbol variants were significant, $\chi^{2}(4)=18.892, p<=0.0008$.

To further examine the data, the Wilcoxon Matched-Pairs Signed-Ranks Test was used to compare participants' preferences on the symbol variants (Table 8). With significant level at $\alpha=0.01$, variant 2-1 was ranked significantly higher when compared to variants 2-2 and 2-5; 
variant 2-4 was ranked significantly higher when compared to variants 2-2 and 2-5. There was no significance between variant 2-1 and variant 2.4. There were no significant differences of variant 2-3 when compared to other variants.

From the comparisons, variants 2-1 and 2-4 appear to be the most preferred symbols to represent the concept of "biometrics in use". Thus, those two symbol variants (Figure 5) were chosen as candidates and were socialized at the ISO/IEC JTC 1/SC 37 WG 6 working group meeting in Hawaii to solicit feedback from ISO members.

Table 8 Comparisons of preference rankings among Symbol Variants

\begin{tabular}{|l|l|l|l|l|}
\cline { 2 - 5 } \multicolumn{1}{c|}{} & \multicolumn{1}{c|}{ Variant 2-1 } & \multicolumn{1}{c|}{ Variant 2-2 } & Variant 2-3 & Variant 2-4 \\
\hline Variant 2-2 & $W=261.50, p<=0.001^{*}$ & & & \\
\hline Variant 2-3 & $W=433.5, p<=0.05$ & $W=437, p<=0.10$ & & \\
\hline Variant 2-4 & $W=597.5, p>0.2$ & $W=356, p<=0.01^{*}$ & $W=462.50, p<=0.20$ & \\
\hline Variant 2-5 & $W=343, p<=0.005^{*}$ & $W=578.5, p>0.2$ & $W=426, p<=0.10$ & $W=261.50, p<=0.001^{*}$ \\
\hline
\end{tabular}

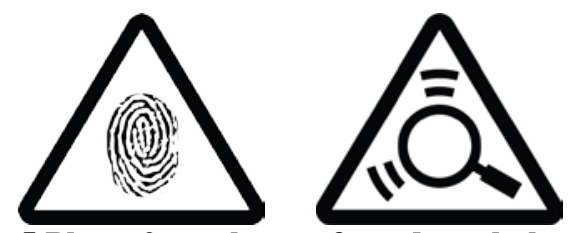

Figure 5 Phase 2 result - preferred symbol variants

The WG6 members were concerned with the symbols being warning signs as these symbols may be perceived by the general public that the use of biometrics could be dangerous or harmful. They recommended developing an informative symbol to communicate to general public the concept that biometrics may be in use in the vicinity surroundings. As discussed earlier, to address the concerns raised by WG6 members, another set of three variants without the triangular border was developed and was evaluated in phase 3.

\subsection{PHASE 3}

\subsubsection{Respondents' Preference Rankings}

Out of the 725 people who took the on-line survey, 16 respondents' data were excluded from data analysis as they did not cast any votes toward the three variants in Question \#5 on the survey (see Appendix B). Partial votes, for example, votes were casted for 1 or 2 variants, were included in data analysis. Total of 709 respondents' data were included in the analyses. The counts of respondents voting for the variants as $1^{\text {st }}, 2^{\text {nd }}$, or $3^{\text {rd }}$ are shown in Figure 6.

As the votes over the three symbol variants were ordinal and related (within participants), the Friedman's analysis of variance by ranks, was used. Differences of preferences across symbol variants were significant, $\chi^{2}(2)=280.930, p=0.0000$. 
To further examine the data, the Wilcoxon Matched-Pairs Signed-Ranks Test was used to compare participants' preferences on the symbol variants. At $\alpha=0.01$, variant 3-1 was ranked significantly higher when compared to variants 3-3 ( $p=0.0000)$; variant 3-2 was ranked significantly higher when compared to variants $3-3(p=0.0000)$. There was no significance between variant 3-1 and variant $3.2(p=0.027)$ at $\alpha=0.01$, but the difference was significant at $\alpha=0.05$, with variant $3-1$ ranked higher than variant 3-2.

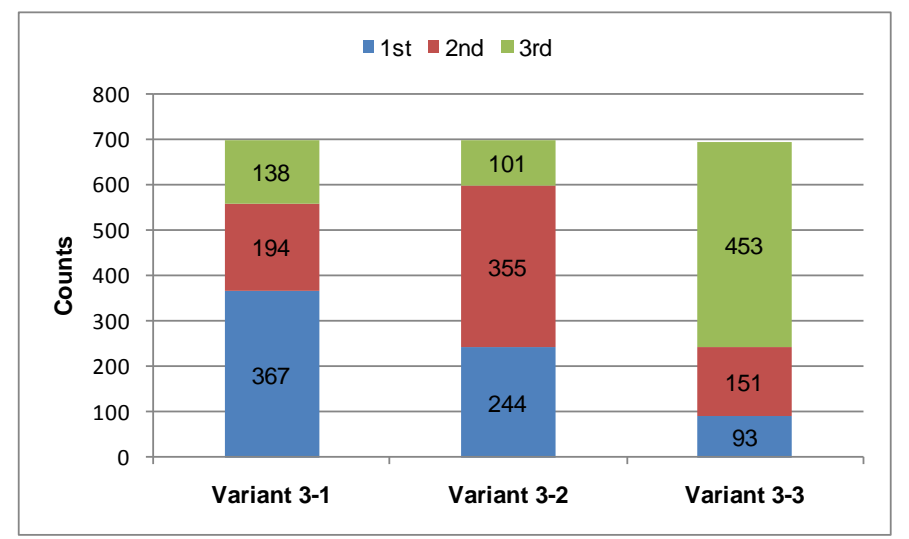

Figure 6 Respondents' votes for the 3 symbol variants

To determine whether the preference rankings were different among different age groups, the Friedman's analysis of variance by ranks was used. Differences in preferences across symbol variants were significant among different age groups, $\chi^{2}(2)=10.75, p=0.0046$, at $\alpha=0.01$. The score rankings within age groups from Friedman test are listed in Table 9. The age group of "75 and over" was the only group with different ranking patterns that the participants in this group preferred variant 3-3, followed by variant 3-1, then variant 3-2; whereas all other age groups gave variant 3-1 top rank, followed by variant 3-2, then variant 3-3. It should be noted that the "75 and over" age group consisted only two participants which represented a very small percentage $(0.28 \%)$ of the survey participants.

Table 9 Score rankings on symbol variants within age groups

\begin{tabular}{|l|c|c|c|}
\cline { 2 - 4 } \multicolumn{1}{c|}{ Age Group } & \multicolumn{3}{c|}{ Vcore Rankings } \\
\hline $18-24(\mathrm{n}=57)$ & 1 & Variant 3-2 & Variant 3-3 \\
\hline $25-34(\mathrm{n}=148)$ & 1 & 2 & 3 \\
\hline $35-44(\mathrm{n}=182)$ & 1 & 2 & 3 \\
\hline $45-54(\mathrm{n}=213)$ & 1 & 2 & 3 \\
\hline $55-64(\mathrm{n}=81)$ & 1 & 2 & 3 \\
\hline $65-74(\mathrm{n}=21)$ & 1 & 2 & 3 \\
\hline 75 and over $(\mathrm{n}=2)$ & 2 & 3 & 3 \\
\hline Not specified $(\mathrm{n}=5)$ & 1 & 2 & 3 \\
\hline
\end{tabular}


To examine whether there were gender differences in ranking the symbol variants, Friedman's analysis of variance by ranks was used. Three respondents who did not specify their genders were excluded from this analysis. There were 198 males and 508 females. Differences in preferences across symbol variants between male and female respondents were not significant, $\chi^{2}(2)=4.000, p=0.1353$, at $\alpha=0.01$.

\subsubsection{Responses on Symbol Clarity}

For each symbol variant, respondents were asked a question: "The symbol clearly describes the concept of biometrics in use.” The question used Likert-scale ratings, with " 1 ” as "Unclear" and "5" as "Completely clear."

A non-parametric analysis, Kruskal-Wallis one-way ANOVA, was used. Differences in the ratings on symbol clarity across symbol variants were significant, $H=455.8078$ (critical value for $\chi^{2}$ with $p<=0.01$ and $2 d f$ is 7.82).

To further examine the data, the Mann-Whitney U test was used to compare participants' opinion ratings on symbol clarity. The medians and the Mann-Whitney $U$ test results are listed in Table 10. With significant level at $\alpha=0.01$, variant 3-1 was rated significantly higher on symbol clarity than variant 3-2 and variant 3-3, and variant 3-2 was rated significant higher than variant 3-3.

Table 10 Medians and comparisons of ratings on symbol clarity

\begin{tabular}{|l|c|c|c|}
\cline { 2 - 4 } \multicolumn{1}{c|}{} & Variant 3-1 & Variant 3-2 & Variant 3-3 \\
\hline Variant 3-2 & $U=320521, p=0.0000$ & & \\
\hline Variant 3-3 & $U=230720, p=0.0000$ & $U=386779.5, p=0.0000$ & \\
\hline Median ( $\mathrm{n}=725)$ & 4 & 3 & 1 \\
\hline
\end{tabular}

\subsubsection{Responses on Symbol I nduced Confusion}

For each symbol variant, respondents were asked a question: "The symbol is confusing." The question used Likert-scale ratings, with " 1 " as "Not confusing” and " 5 " as "Completely confusing."

A non-parametric analysis, Kruskal-Wallis one-way ANOVA, was used. Differences in the ratings on symbol clarity across symbol variants were significant, $H=393.5126$ (critical value for $\chi^{2}$ with $p<=0.01$ and $2 d f$ is 7.82).

Table 11 Medians and comparisons of ratings on symbol induced confusion

\begin{tabular}{|l|c|c|c|}
\cline { 2 - 4 } \multicolumn{1}{c|}{} & Variant 3-1 & Variant 3-2 & Variant 3-3 \\
\hline Variant 3-2 & $U=313990, p=0.0000$ & & \\
\hline Variant 3-3 & $U=412485, p=0.0000$ & $U=374825.5, p=0.0000$ & \\
\hline Median ( $\mathrm{n}=725)$ & 1 & 2 & 3 \\
\hline
\end{tabular}

To further examine the data, the Mann-Whitney U test was used to compare participants' opinion ratings on the degree that symbols appear to be confusing to the participants. The 
medians and the Mann-Whitney $\mathrm{U}$ test results are listed in Table 11 . With significant level at $\alpha=0.01$, variant 3- 1 was rated significantly as being less confusing than variant 3-2 and variant 3-3, and variant 3-2 was rated significant less confusing variant 3-3.

\subsubsection{Responses on Confidence in Symbol Recognition}

For each symbol variant, respondents were asked a question: "How confident are you that you would recognize the symbol as biometrics in use if you saw it in the future?" The question used Likert-scale ratings, with " 1 " as "Not confident" and " 5 ” as "Completely confident."

A non-parametric analysis, Kruskal-Wallis one-way ANOVA, was used. Differences in the ratings on symbol clarity across symbol variants were significant, $H=377.8455$ (critical value for $\chi^{2}$ with $p<=0.01$ and $2 d f$ is 7.82).

To further examine the data, the Mann-Whitney U test was used to compare participants' opinion ratings on confidence in recognizing symbol in the future. The medians and the Mann-Whitney U test results are listed in Table 12 . With significant level at $\alpha=0.01$, variant 3-1 was rated significantly higher on confidence in recognizing the symbol in the future than variant 3-2 and variant 3-3, and variant 3-2 was rated significant higher than variant 3-3.

Table 12 Medians and comparisons of ratings on confidence in symbol recognition

\begin{tabular}{|l|c|c|c|}
\cline { 2 - 4 } \multicolumn{1}{c|}{} & Variant 3-1 & Variant 3-2 & Variant 3-3 \\
\hline Variant 3-2 & $U=320109.5, p=0.0000$ & & \\
\hline Variant 3-3 & $U=409744, p=0.0000$ & $U=368788.5, p=0.0000$ & \\
\hline Median (n=725) & 4 & 3 & 2 \\
\hline
\end{tabular}

\subsubsection{Discussions}

From the results, variant 3-1 (Figure 7(a)) is the best candidate for representing the concept of "biometrics in use" as it received highest preference ranking, was perceived as clearer and less confusing and respondents felt more confident to recognize the symbol in the future. However, some respondents shared their concerns that, although the symbol is well recognized and understood, it is too specific representing only single modality which may limit its capability to be generalized in the situation where multiple modalities are in use. For example, one respondent stated "I do think it clearly displays biometrics as a symbol but it does not define the whole spectrum of what can be used." Another respondent commented "This symbol gives the impression of fingerprints only whereas the other symbols suggests more." One respondent also stated "I associate this symbol with a fingerprint, and nothing else. This symbol I have already seen for things requiring a simple fingerprint, and I would not associate it with the wider scope of biometrics.” 


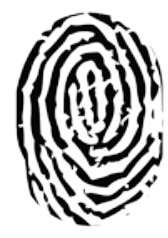

(a)

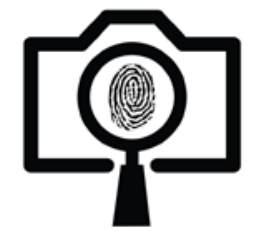

(b)

Figure 7 Phase 3 result - two symbol candidates for "Biometrics in Use"

Variant 3-2 (Figure 7(b)) seems to be another plausible candidate as its preference ranking was not significantly different from variant 3-1. And, it was rated second consistently regarding its clarity, induced confusion, and respondents' confidence in recognizing it in the future. Respondents who ranked it first pointed out that it covers a broader spectrum of biometric modalities. One respondent commented "[variant 3-2] symbolizes everything for biometrics." Another respondent stated "Biometrics is a finger print, eye scan, or a face photo and this symbol is the best!" Some respondents who ranked it second were mainly concerned with the small size of the fingerprint image in the center that may cause recognition problems when the symbol is reduced to smaller sizes, for example, as an icon on a computer screen. One respondent commented "As long as it was good size it would work. The smaller the worse it becomes." Another respondent stated "this symbol captures biometrics but if the symbol is minimized, I [can] barely see the fingerprint.” Another noted "My only reason for not voting this icon in the \#1 position is due to the fingerprint being too small for some to easily view. If the fingerprint were larger/easier to identify, I'd use this as the first choice."

\section{CONCLUSION}

From the results, two variants are plausible candidates to be proposed to the ISO/IEC JTC 1/SC 37 WG 6 for consideration to represent the concept of "biometrics in use." It is recommended to use variant 3-1, i.e. fingerprint image (Figure 7(a)), when only the fingerprint collection is in use. When multiple modalities are in use, variant 3-2 (Figure 7(b)) is to be used.

When the symbols are to be used as public information symbols, they can be reproduced in any color and be enclosed with borders (ISO 7001 [1]). Examples of how the two symbols can be presented as public information symbols with optional supplementary text are demonstrated in Figure 8 (a) and (b). 


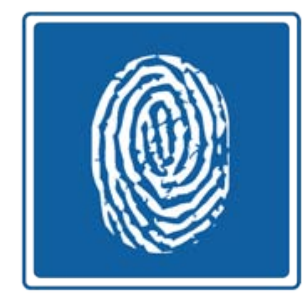

Biometrics in Use

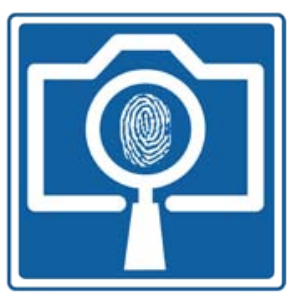

Biometrics in Use

(a)

(b)

Figure 8 Examples of public information symbols for "Biometrics in Use"

If the symbols were to be used as alerting symbols for certain situations, such as in workplaces and public areas, design principles outlined in ISO 3864-1 [3] should be followed. A warning sign shall use yellow safety color, black contrast color, and be displayed in an equilateral triangle. Examples of the symbols for "biometrics in use" used as alerting signs optional supplementary text are demonstrated in Figure 9 (a) and (b).

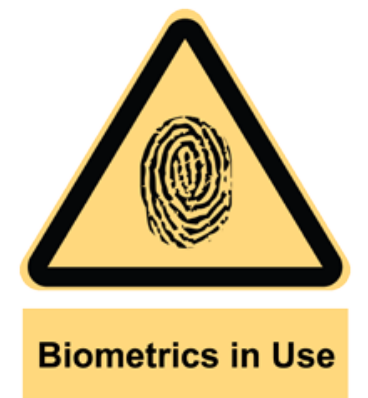

(a)

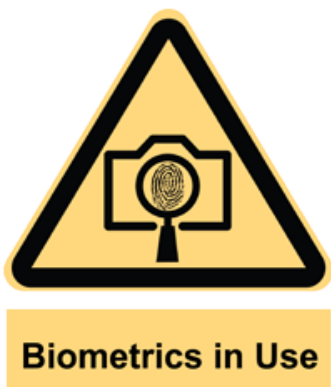

(b)

Figure 9 Examples of alerting symbols for "Biometrics in Use"

Some future research is needed before finalizing the symbol variants for representing the concept of "Biometrics in Use." Firstly, based on comments made by the survey respondents, the fingerprint image in both variants may need to be simplified to address respondents' concerns on legibility and also to help in image scaling, especially when reducing the size of the symbols is necessary. Secondly, these symbols need to be evaluated in an operational environment where the symbols will be used to ensure that the general public is informed of "biometrics in use" by the symbol. Thirdly, the symbols should be evaluated in other countries to understand if there will be any cultural implications. 


\section{REFERENCES}

[1] International Organization for Standards. ISO 7001 Graphical symbols - Public information Geneva, Switzerland: Author, (2007).

[2] International Organization for Standards. ISO/IEC 13251 Collection of graphical symbols for office equipment Geneva, Switzerland: Author, (2004).

[3] International Organization for Standards. ISO 3864-1 Graphical symbols - Safety colours and safety signs - Part 1: Design principles for safety signs in workplaces and public areas Geneva, Switzerland: Author, (2002).

[4] International Organization for Standards. ISO 7010 Graphical symbols - Safety colours and safety signs - Safety signs used in workplace and public areas Geneva, Switzerland: Author, (2003).

[5] International Organization for Standards. ISO 7010 Graphical symbols -Safety colours and safety signs - Safety signs used in workplace and public areas, Amendment 1 Geneva, Switzerland: Author, (2006).

[6] International Organization for Standards. ISO 7010 Graphical symbols -Safety colours and safety signs - Safety signs used in workplace and public areas, Amendment 2 Geneva, Switzerland: Author, (2007).

[7] International Organization for Standards. ISO 7010 Graphical symbols -Safety colours and safety signs - Safety signs used in workplace and public areas, Amendment 3 Geneva, Switzerland: Author, (2007).

[8] International Organization for Standards. ISO 7010 Graphical symbols -Safety colours and safety signs - Safety signs used in workplace and public areas, Amendment 4 Geneva, Switzerland: Author, (2009).

[9] International Organization for Standards. ISO 6309 Fire protection - safety signs Geneva, Switzerland: Author, (1987).

[10] International Organization for Standards. ISO 20712-1 Water safety signs and beach safety flags - Part 1: Specifications for water safety signs used in workplaces and public areas Geneva, Switzerland: Author, (2008). 


\section{APPENDIX A: PHASE 3 - EMAIL TO INVITE SURVEY PARTICIPANTS}

\section{Dear Survey Participant,}

The international community is voting on symbols for biometric systems such as fingerprint, eye (iris) scans, and face photo. The United States National Institute of Standards and Technology (NIST) is working with the international community to identify symbols that can be used anywhere in the world with biometric systems. We are currently voting on a symbol to inform people that biometrics are being collected.

In general symbols are learned. For example you learned that a stop sign is red and has 8 sides and that a caution sign is a yellow triangle. You would not recognize these without someone teaching you.

The symbol we are voting on will need to be learned. The symbol should also be easy to remember and remind you of biometrics. It will be used to identify all types of biometrics including eye, face photo, and fingerprint.

We need your vote. Please help us find the biometric symbol by completing a short questionnaire. It will only take 5 minutes.

Simply click on the link below or copy the link into your Internet browser. The link will only be available for the next 10 days.

http://control.nist.gov/biometrics/cgi/biometrics_survey.cgi

Thank you

Sincerely,

The NIST Team, biosymbol@nist.gov 


\section{APPENDIX B: PHASE 3 - EXAMPLE OF BIOMETRICS SYMBOL SURVEY}

This example shows a variant (out of six variants) of the survey as it was presented on the internet.

\section{Biometric Symbol Survey}

It will take about 5 minutes to complete the following survey. Thank you for your participation.

1. Your Age: (Select one)

2. Gender: $\bigcirc$ Male $\bigcirc$ Female

3. Ethnic background: (Select one)

If your ethnic background is not listed above, please specify

4. Your Job: (Select one)

If your job is not listed above, please specify:

5. Below, there are 3 symbols for biometrics in use for you to vote on. Please select a number for each symbol:

- Select "1" for the symbol that you like the most, that is the easiest to remember and reminds you the most of biometrics.

- Select "2" for the symbol that you like less.

- Select "3" for the symbol that you like the least.

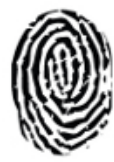

(Vote)

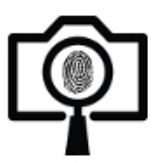

(Vote) $\vee$

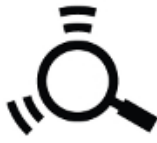

(Vote)
Reset Votes 6a. The symbol

Unclear

O

1

clearly describes the concept of biometrics in use.

$\begin{array}{cc}\text { Somewhat clear } & \text { Clear } \\ 0 & \bigcirc \\ \mathbf{2} & \mathbf{3}\end{array}$

Completely clear

○ 
6b. The symbol

$\begin{array}{ccccc}\text { Not confusing } & \text { Somewhat confusing } & \text { Confusing } & \text { Very confusing } & \text { Completely confusing } \\ 0 & 0 & 0 & 0 & 0 \\ \mathbf{1} & \mathbf{2} & \mathbf{3} & \mathbf{4} & \mathbf{5}\end{array}$

6c. How confident are you that you would recognize the symbol

$\begin{array}{ccccc}\text { Not confident } & \text { Somewhat confident } & \text { Confident } & \text { Very confident } & \text { Completely confident } \\ 0 & 0 & 0 & \bigcirc & 0 \\ \mathbf{1} & \mathbf{2} & \mathbf{3} & \mathbf{4} & \mathbf{5}\end{array}$

6d. Do you have any additional comments on the symbol ?

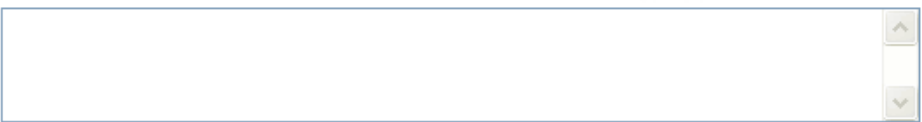

7a. The symbol clearly describes the concept of biometrics in use

$\begin{array}{ccccc}\text { Unclear } & \text { Somewhat clear } & \text { Clear } & \text { Very clear } & \text { Completely clear } \\ 0 & 0 & 0 & 0 & 0 \\ \mathbf{1} & \mathbf{2} & \mathbf{3} & \mathbf{4} & \mathbf{5}\end{array}$

7b. The symbol is confusing

$\begin{array}{ccccc}\text { Not confusing } & \text { Somewhat confusing } & \text { Confusing } & \text { Very confusing } & \text { Completely confusing } \\ 0 & 0 & 0 & 0 & 0 \\ \mathbf{1} & \mathbf{2} & \mathbf{3} & \mathbf{4} & \mathbf{5}\end{array}$

7c. How confident are you that you would recognize the symbol as biometrics in use if you saw it in the future?

$\begin{array}{ccccc}\text { Not confident } & \text { Somewhat confident } & \text { Confident } & \text { Very confident } & \text { Completely confident } \\ 0 & 0 & 0 & 0 & 0 \\ \mathbf{1} & \mathbf{2} & \mathbf{3} & \mathbf{4} & \mathbf{5}\end{array}$


8a. The symbol clearly describes the concept of biometrics in use.

Unclear

O

1
Somewhat clear

O
Clear

0
Very clear

○

4
Completely clear

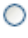

8b. The symbol

Not confusing

O

1
Somewhat confusing

O
Confusing

O

3
Very confusing

○
Completely confusing

0

8c. How confident are you that you would recognize the symbol

$\begin{array}{ccccc}\text { Not confident } & \text { Somewhat confident } & \text { Confident } & \text { Very confident } & \text { Completely confident } \\ 0 & 0 & 0 & 0 & 0 \\ \mathbf{1} & \mathbf{2} & \mathbf{3} & \mathbf{4} & \mathbf{5}\end{array}$

8d. Do you have any additional comments on the symbol ?

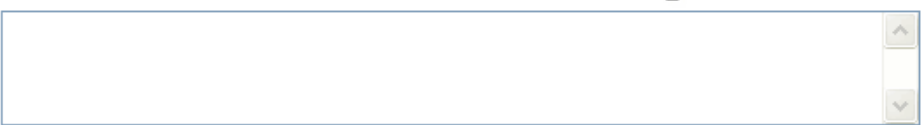

9. Have you ever had your biometrics captured before? $\bigcirc$ Yes $\bigcirc$ No

If yes, check all that apply:

$\square$ Fingerprinted with ink/paper

Eye Scan

Fingerprinted electronically

Face Image

Palm Print

Voice

$\square$ Hand Geometry

\section{Submit Reset}

NOTE: This survey contains collection of information requirements subject to the Paperwork Reduction Act. Notwithstanding any other provisions of the law, no person is required to respond to, nor shall any person be subject to penalty for failure to comply with, a collection of information subject to the requirements of the Paperwork Reduction Act. The estimate response time for this survey is 15 minutes. The response time includes the time for reviewing instructions, searching existing data sources, gathering and maintaining the data needed, and completing and reviewing the collection of information. Send Comments regarding this estimate or any other aspects of this collection of information, including suggestions for reducing the length of this questionnaire, to the National Institute of Standards and Technology, Attn., Mary Theofanos, via email.

The OMB number for this survey is 0693-0043, expiring on 7/31/2009. 
